\title{
Activity of an Identified Histaminergic Neuron, and its Possible Role in Arousal of Feeding Behavior in Semi-Intact Aplysia
}

\author{
Klaudiusz R. Weiss, ${ }^{*}$ Hillel J. Chiel, ${ }^{1}$ U. Koch, ${ }^{2}$ and Irving Kupfermann $\dagger$ \\ Center for Neurobiology \& Behavior, The New York State Psychiatric Institute, New York, New York 10032, \\ Departments of †Physiology, "Anatomy and Cell Biology, and *†Psychiatry, ${ }^{*}+$ Columbia University College of \\ Physicians and Surgeons, and *School of Dental and Oral Surgery, New York, New York 10032
}

\begin{abstract}
The possible functions of histaminergic neuron $\mathrm{C} 2$ were studied in an isolated head preparation from which it was possible to obtain intracellular recordings while the buccal mass exhibited feeding-like responses. Application of food to the lips of the isolated head preparation elicited rhythmic buccal movements that appeared to be ingestion responses, since they moved seaweed into the buccal cavity and towards the esophagus, and their frequency and regularity was similar to the ingestion responses studied in a group of intact animals.

The ingestion responses of the buccal mass consisted of 2 main phases of movement of the radula from a middle rest position: (1) forward and return to rest, and (2) backward and return to rest. The relative magnitudes and timing of these 2 phases were variable. Intracellular recordings from $\mathrm{C} 2$ in the isolated head revealed that $\mathrm{C} 2$ is silent when the buccal mass is quiescent, but that it can be excited into spike activity, either by mechanical stimulation of the perioral zone or by chemostimulation that results in rhythmic movement. C2 fires a burst of spikes in phase with each protraction-retraction cycle, and, if the movements continue, $\mathrm{C} 2$ fires even when the eliciting stimulus has been removed. Activity of the cell was usually preceded by fast depolarizing responses that appeared to be blocked axon spikes. The evidence suggests that $\mathrm{C} 2$ is part of a positive feedback loop that may help maintain the persistence of arousal of feeding behavior beyond the time that food stimuli are removed.
\end{abstract}

The metacerebral cell (MCC) of Aplysia mediates a number of behavioral effects associated with an arousal state induced by food (Weiss et al., 1982). One source of excilatory input to the MCC is the histaminergic neuron C2 (Weiss et al., 1986a), which suggests that $\mathrm{C} 2$ may function as part of a central arousal system that serves to sustain the arousal state. An important step in exploring this hypothesis is determining the activity of $\mathrm{C} 2$ during actual feeding behavior. In previous studies of the role of the MCC in normal feeding, we took advantage of the large size of the peripheral axons of this neuron to record its activity in free-moving, intact animals (Kupfermann and Weiss, 1982). However, the peripheral axons of $\mathrm{C} 2$ are relatively small and difficult to record from. Therefore, to study the role of $\mathrm{C} 2$ in food-arousal and other behavioral functions, we developed a

Received Oct. 22, 1985; revised Feb. 18, 1986; accepted Feb. 20, 1986

We wish to thank Drs. T. J. Carew, V. J. Castellucci, G. Clark, E. R. Kandel, and J. Koester for their comments and discussion. This work was supported in part by PHS Grants MH35564, GM320099, and RSDA MH00304

Correspondence should be addressed to Dr. I. Kupfermann, Center for Neurobiology \& Behavior, 722 West 168 th Street, Psychiatric Institute Annex-8th floor, New York, NY 10032.

' Present address: Bell Laboratories, 600 Mountain Avenue, Murray Hill, NJ 07974.

${ }^{2}$ Present address: Fachbereich Biologie der Universitat, D-6750 Kaiserlautern, West Germany.

Copyright (C) 1986 Society for Neuroscience $0270-6474 / 86 / 082403-13 \$ 02.00 / 0$ semi-intact preparation that permits intracellular recording while the animal performs behavioral responses. Although semi-intact preparations of Aplysia readily exhibit defensive, escape, and withdrawal responses (Carew and Kandel, 1977; Hening et al., 1979; Jahan-Parwar and Fredman, 1978; Kupfermann and Kandel, 1969), appetitive responses such as feeding are exceedingly difficult to obtain. In previous work, a semi-intact preparation that exhibited movements of the buccal mass was developed (Koch et al., 1984), but the possible relationship of these movements to those that occurred during normal feeding was not clear. In the present study, we report the use of an improved methodology to obtain an isolated head preparation whose behavior exhibits a number of similarities to feeding behavior in the intact animal. Using this preparation, we have found that $\mathrm{C} 2$ is phasically active during feeding-like responses. Activity of $\mathrm{C} 2$ outlasts the initiating stimulus, and we provide evidence that the cell may help to maintain arousal during feeding behavior.

\section{Materials and Methods}

\section{Apparatus used for the isolated head preparation}

The chamber in which the preparation was mounted could be connected to 3 perfusion sources: an input source, a circulation circuit, and an arterial perfusion circuit (Fig. 1). The input source consisted of a carboy containing artificial seawater mounted on a shelf 2 feet above the chamber, whose outflow was cooled by glass and metal heat exchangers, connected to a Lauda K-4/R cooler (Brinkmann), before reaching the chamber. The circulation circuit utilized a piston or peristaltic pump that drew water out of the chamber, forced it through a charcoal/glass wool filter, passed it through a heat exchanger to cool it, and returned it to the chamber. The perfusion circuit consisted of a Marriote bottle, which aerated the water and could be adjusted in height to control perfusion pressure, which was maintained at approximately $25 \mathrm{~mm}$ of water, measured at the chamber. Before the perfusion fluid was sent into the buccal artery, it was passed through a heat exchanger to be cooled and then into a bubble separator. From there, the perfusion was sent to a cannula that was inserted into the buccal artery. The bubble separator was connected to a vertical tube, open to the air and attached in parallel to a pressure transducer (Harvard Apparatus), which provided a measure of the flow resistance of the preparation.

\section{Description of the isolated head preparation, and} electrophysiological methods

The isolated head of Aplysia never exhibited feeding behavior unless prepared in a particular way. The preparation we used was based on modifications of a previous preparation (Koch et al., 1984) and was developed after considerable trial and error. We have not determined in a controlled way what conditions are absolutely essential in order for the preparation to show feeding behavior, but our experience has indicated that of particular importance are (1) temperature, (2) arterial perfusion at the correct pressure, and (3) proper positioning of the buccal mass and head tissue.

Before the experiment, animals were maintained in artificial seawater in a 100 gallon tank at $15^{\circ} \mathrm{C}$. However, for $48 \mathrm{hr}$ just prior to the 


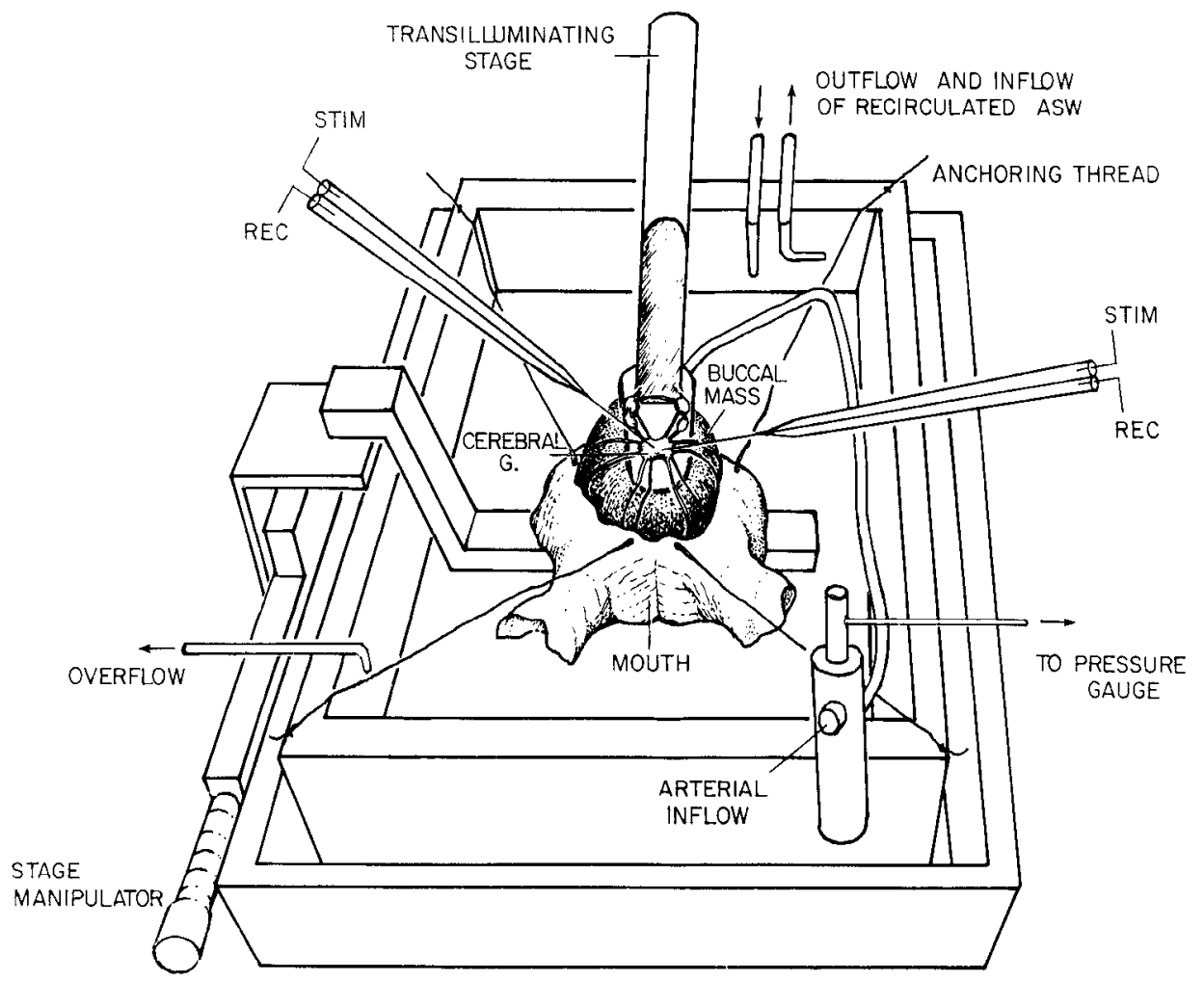

Figure 1. A semidiagrammatic representation of the preparation and apparatus used for simultaneous recording of feeding movements and for intracellular recording and stimulation.

preparation of the isolated head, animals were placed in a 15 gallon tank that contained filtered and aerated seawater kept $7^{\circ} \mathrm{C}$ warmer than the water in the home tank. Animals were not fed while they were maintained in the warm-water tank, but were tested for feeding responses on the day they were used for the isolated head preparation. Only those animals that showed vigorous biting responses were used for experiments. Prior to dissection, animals were immobilized with an injection of isotonic magnesium chloride ( $50 \% \mathrm{vol} / \mathrm{wt}$; Kupfermann, 1974a). During dissection, animals were maintained in a solution of $50 \%$ seawater and $50 \%$ isotonic magnesium chloride. After an animal was fully relaxed, an incision was made on the dorsal surface behind the rhinophores. All ganglia were retained, but the peripheral nerves of the abdominal and the pleural-pedal ganglia were cut. In addition, branches of the cerebral nerves innervating the cut tissue edges were cut, since this appeared to decrease the likelihood that the preparation would "sensitize" and not feed after the high magnesium ion solution was removed. Because preliminary studies suggested that the isolated head preparation fed erratically if large parts of the gut were left attached to the buccal mass, the gut was removed at the level of the esophagus. The head was cut from the rest of the animal at the level of the rhinophores (dursally) and slightly caudal to the beginning of the foot (ventrally). Two silk threads were inserted into the dorsal surface of the head tissue, and 2 were inserted into the ventral surface. The threads were subsequently used for positioning the head in the chamber (see Fig. 1). The buccal artery was dissected free of connective tissue at the level of the pleural-pedal ganglia. Once the dissection was complete, the head was transferred to the experimental chamber. The cannula in the chamber was flushed with seawater to eliminate air bubbles, and the buccal artery was gently led over the tip of the cannula and tied in place. The preparation was then positioned on a small pedestal using the threads that had previously been inserted into the skin. A small lucite platform was placed underneath the cerebral ganglion, which was then pinned to a layer of soft plastic (Sylgard) that covered the substage. The substage was attached to a lucite rod that could be illuminated from the top and that provided transillumination of the ganglion.

Once the dissection of the sheath of the cerebral ganglion was completed, the recirculation of seawater through the chamber was stopped, and the chamber clamped to a stage on the electrophysiology setup. Fresh, cooled $\left(17^{\circ} \mathrm{C}\right)$ seawater was added to the bath at a rate of at least $12 \mathrm{ml} / \mathrm{min}$ through the input circuit. The perfusion cannula that had been inserted into the buccal artery was attached to the arterial perfusion circuit, and the preparation was perfused with seawater at a pressure of
$25 \mathrm{~mm}$ of water, as measured at the bubble separator; the resulting rate

The animal was tested after approximately $60 \mathrm{~min}$, when the excess magnesium had been washed out of the preparation and the chamber. The temperature of the chamber was maintained at $17 \pm 1^{\circ} \mathrm{C}$. Intracellular recordings were made with double-barreled microelectrodes filled with 2 m potassium citrate. Standard electrophysiological methods were used in this study (Rosen et al., 1982).

\section{Methods of observing biting, swallowing, and rejection in intact animals}

In order to observe and quantitate biting and swallowing behavior, five intact animals (100-200 gm) were presented with a series of seaweed stimuli (Laver). Rhythmic biting responses were elicited by touching a $1 \mathrm{~cm}^{2}$ piece of seaweed to the perioral zone. At the onset of protraction of the radula, the seaweed was moved to the side of the lips so that the animal could not grasp it. Upon retraction of the radula, the seaweed was immediately placed again at the perioral zone. On trials in which rhythmic swallowing movements were elicited, the animal was given a strip of seaweed $10 \mathrm{~cm}$ in length. Two widths of strips were presented, wide $(2 \mathrm{~cm})$ and narrow $(1 \mathrm{~cm})$. Bites were indicated on a chart recorder at the onset of the retraction of the radula, whereas swallows were indicated at the onset of a inward movement of the strip of seaweed. Each of 5 animals was given 4 runs of biting and of swallowing with narrow and wide strips. To study rejection movements, 5 additional animals (100-200 gm) were used. A $15 \mathrm{~cm}$ length of silastic tubing (medical grade, no. 5, 0.040 in. $\times 0.085$ in.; Storz Instruments, St. Louis, $\mathrm{MO}$ ) was partially inserted into the esophagus. Insertion of the tubing was aided by first initiating biting responses with a small piece of seaweed. To facilitate observation of the movement of the tubing, it was marked with regularly spaced black dots, and $2 \mathrm{~cm}$ of each end were marked with red. Each outward movement of the tube was indicated manually on a chart recorder.

\section{Results}

Behaviors involving the buccal mass of Aplysia

It has previously been suggested (Kupfermann, 1974b) that the buccal mass is involved in at least 3 distinct behaviors: biting, 
swallowing, and rejection. Recently it has been suggested that the buccal mass of the mollusk, Pleurobranchaea, is involved in at least 5 different behaviors, including regurgitation and gill grooming (Croll et al., 1985a; McClellan, 1982). In order to provide some insight into the nature of the movements of the buccal mass in the isolated head preparation, we have made additional behavioral observations of normal feeding in freemoving Aplysia.

We have confirmed our previous finding of a distinction between biting and swallowing movements. Biting is elicited by stimulation of the perioral zone and the adjacent tissue around the mouth. During biting, the radula extends out of the mouth, which opens wide at the peak of protraction. If a strip of food is presented and the animal is permitted to ingest it, there occur a series of swallows consisting of rhythmic movements that draw the strip into the buccal cavity in a saltatory fashion. Unlike biting, swallowing movements are not associated with wide opening of the mouth, and the radula cannot be seen. To determine if the rates of biting and swallowing were different, repetitive biting and swallowing movements were induced in 5 animals. Biting was triggered by stimulating the mouth with seaweed. The seaweed was moved to the side whenever a bite occurred so that the animal could not ingest the food (see Materials and Methods). Swallowing was elicited by allowing the animal to ingest a strip of seaweed, either narrow (1 cm wide) or wide ( $2 \mathrm{~cm}$ wide).

We found that, for each of the 5 animals studied, there were small but consistent differences between the rates of swallowing movements and those of biting movements (Fig. $2 A$ ). Furthermore, the rate of swallowing was dependent on the width of the strip of seaweed that was swallowed. For each animal, the interresponse times for the swallowing of wide pieces were longer than those for narrow pieces. The average rate at which an animal swallowed a wide strip of seaweed was lower than its rate of biting, whereas its rate of swallowing of narrow strips of seaweed was higher than its rate of biting (Fig. $2, A, C$ ). These findings support the concept that biting and swallowing are distinct behaviors mediated by the buccal mass, and that differences in the properties of the ingested food can affect the motor program for swallowing. It should be noted that, for the first response to a strip, or when an animal was given a single piece of seaweed instead of a strip, an initial bite was elicited, followed by a swallow. We refer to this sequence as a "bite-swallow."

Rejection movements were studied in 5 additional animals. Rejection was elicited by stimulating animals with food and inserting a silastic tube down the esophagus during biting movements. Initially, the tube was swallowed and it moved into the animal, similar to a strip of seaweed. After 1 to 5 swallows, the tube was rejected by means of rhythmic responses that resulted in its moving out of the buccal cavity. The mean interresponse time for rejection movements was considerably greater than the interresponse time for either biting or swallowing of narrow or wide strips (Fig. 2A). Furthermore, unlike swallowing or biting, rejection movements were often highly irregular, and the standard deviation for rejection interresponse times was much larger than that for biting or swallowing interresponse times (Fig. $2 B$ ).

In their natural habitat, Aplysia appear to exhibit a form of grazing behavior, during which the animals locomote with their mouths against the substrate, while rhythmic radula movements occur (Kupfermann and Carew, 1974). This type of behavior has also been seen in starved animals placed in a lucite chamber, which permits viewing of the mouth from underneath. The rhythmic radula movements in these instances resemble those observed during biting responses elicited in quiescent animals in a head-up (food-aroused) posture.

We have attempted to induce regurgitation by injecting potentially noxious fluids into the crop (liquid Ivory soap, dilute acid, Fast green dye) by means of a cannula that the animal was

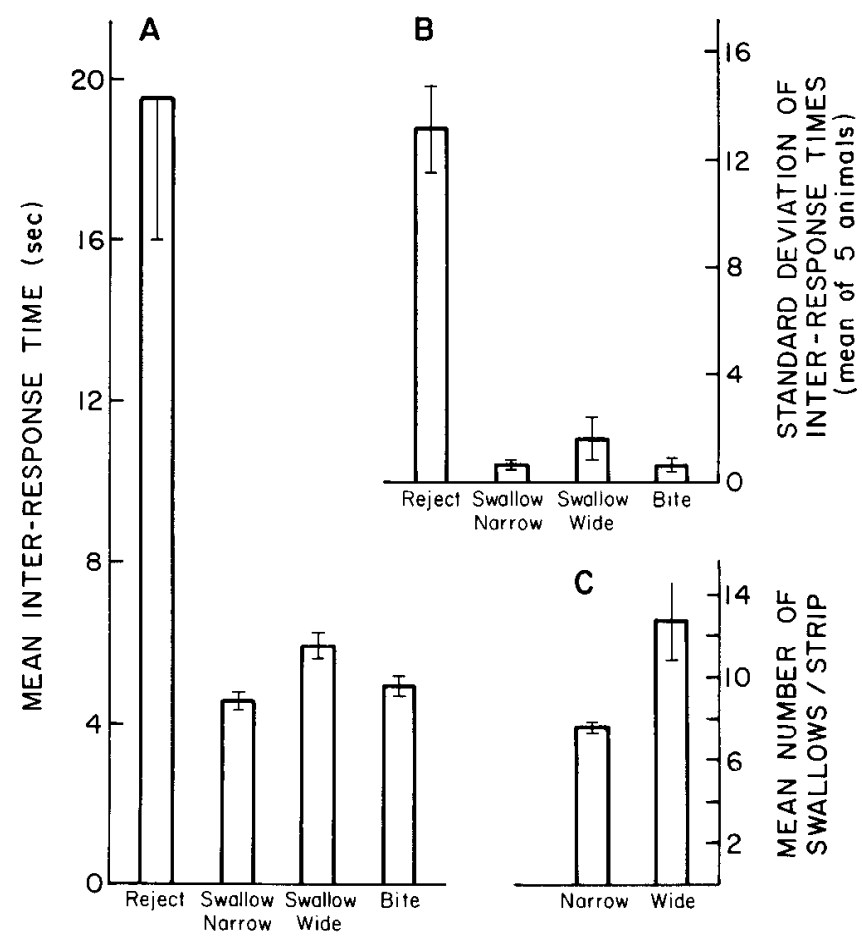

Figure 2. Parametric features of biting, swallowing, and rejection. Five animals were each presented with 4 stimulus conditions: (1) They were induced to swallow a silastic tube and each outward movement was scored as a rejection response. They were given a $(2)$ narrow $(1 \mathrm{~cm})$ or (3) wide $(2 \mathrm{~cm})$ strip of seaweed and each inward movement was scored as a swallow. (4) They were continuously presented with seaweed but were not permitted to ingest it; each protraction was scored as a bite. $A$, Mean time ( $\pm \mathrm{SE})$ between rejection responses, bites, and swallows of narrow or wide strips. $B$, Standard deviation $( \pm \mathrm{SE})$ of the mean interresponse times for rejections, swallows, and bites. For each animal, and for each measure, the standard deviation of interresponse times was determined. Graph shows the mean values (bars) of these standard deviations for 5 animals. $C$, Mean number $( \pm \mathrm{SE})$ of swallows needed to ingest either a narrow or wide strip of seaweed.

made to swallow. In no instance have we observed that these substances elicited rhythmic buccal activity that resulted in ejection of the fluid. The soap solution gradually leaked from the mouth, but this was not associated with observable movements of the body wall of the animal, and was probably due to passive leakage.

Although the buccal mass of Pleurobranchaea has been reported to be involved in gill grooming (McClellan, 1982), we have never observed gill grooming in Aplysia, either in laboratory tanks or in field studies in California (Kupfermann and Carew, 1974) and Israel (Susswein et al., 1984b; A. J. Susswcin and I. Kupfermann, unpublished observations).

\section{Relationships of buccal mass movements in the semi-intact preparation to those of normal feeding}

In order to relate the function of $\mathrm{C} 2$ to feeding, we used a semiintact preparation consisting of the isolated head in which the buccal artery was perfused in order to provide pressure and fluid flow to the lips and buccal mass (see Materials and Methods). This preparation was modified from that described previously by Koch et al. (1984), and produced a more consistent pattern of movements of the buccal mass in response to seaweed stimuli applied to the perioral region. A problem with preparations of this sort is that it is difficult to relate the behavior that occurs to the behavior seen in intact animals. Compounding this prob- 


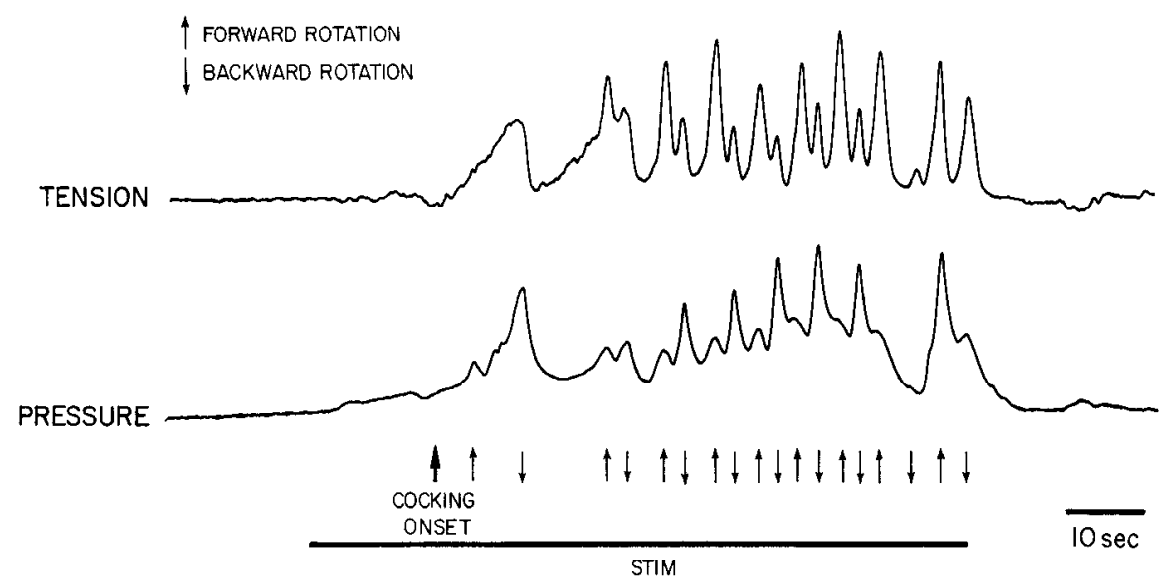

Figure 3. Two methods of simultaneously monitoring feeding behavior of the isolated head preparation. Upper trace shows recording obtained by monitoring tension by means of a transducer attached to the buccal muscle covering the radula sac. Lower trace shows feeding behavior measured by monitoring changes in the pressure of seawater perfusing the buccal mass. Since the inflow was maintained at constant pressure (see Materials and Methods), changes of the pressure in the bubble separator directly reflected changes of the arterial blood flow and resistance of the buccal mass. Arrows in this figure and subsequent figures indicate the peak of forward (up arrow) or backward (down arrow) movement of the buccal mass. Since these were recorded manually on the basis of observations, the time of the arrows includes the reaction time of the observer, and is somewhat delayed relative to the actual movement. In addition, some movements, particularly weak ones, were not indicated.

lem is the fact that quite different behaviors may utilize similar muscles and patterns of muscle contraction. The behavioral studies presented above indicate that the buccal mass of Aplysia is involved in only 2 main classes of behavior: ingestive responses and egestive responses. Ingestive responses occur at regular periods and consist of 2 subclasses, biting and swallowing. Egestive responses tend to occur with an uneven period, and the mean time between responses is long compared to that of ingestive responses. The evidence presented below suggests that the buccal mass movements we observed in the semi-intact preparation were primarily related to ingestive behaviors and not rejection.

The isolated head preparations used in the present studies often displayed spontaneous buccal mass movements. The spontancous activity was irregular and occurred at a low rate relative to food-evoked activity. On the other hand, when seaweed was applied to the perioral zone, it elicited a stereotypic sequence of movements of the buccal mass. Small pieces (less than $1 \mathrm{~cm}^{2}$ ) of seaweed (Laver) were used, and feeding was not evoked unless the seaweed contacted the perioral zone. Thus it is unlikely that feeding was the result of exposure of the nervous system to dissolved substances from the seaweed. The movements were monitored in 2 ways. A strain gauge, attached to the muscle sheath overlying the radula sac, directly recorded forward and backward movement; a pressure transducer indicated the perfusion pressure of the buccal aorta and indirectly recorded movements, since muscle contractions and movements altered the resistance of fluid flow through the tissue. In Figure 3, a simultaneous rccording of pressure and tension of the buccal mass is shown. Since both recordings gave parallel indications of movements, we chose, in most experiments, to record buccal activity using the pressure method. This method is very sensitive, and, since it is noninvasive, it reduces the chance that the animal will sensitize and cease responding to food. Our recordings were usually supplemented by visual observation, which allowed us to determine the direction of movement of the buccal mass.

When food was applied to the lips of the preparation, after a
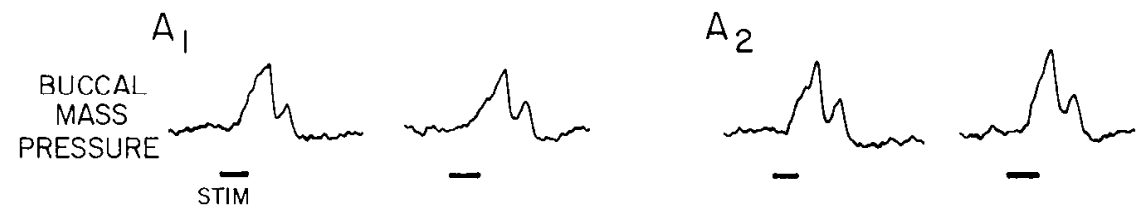

Figure 4. Two types of response to food stimuli in the isolated head preparation. In these records, the cocking phase was fused with protraction. $A_{I}$ and $A_{2}$ are from 2 different preparations. Comparison of the 2 records within $A_{1}$ and $A_{2}$ shows the reproducibility of behavior within an animal. $A$, Brief stimulation produced single bites. $B$, Somewhat more prolonged stimulation produced a bout of feeding movements that outlasted the stimulus.

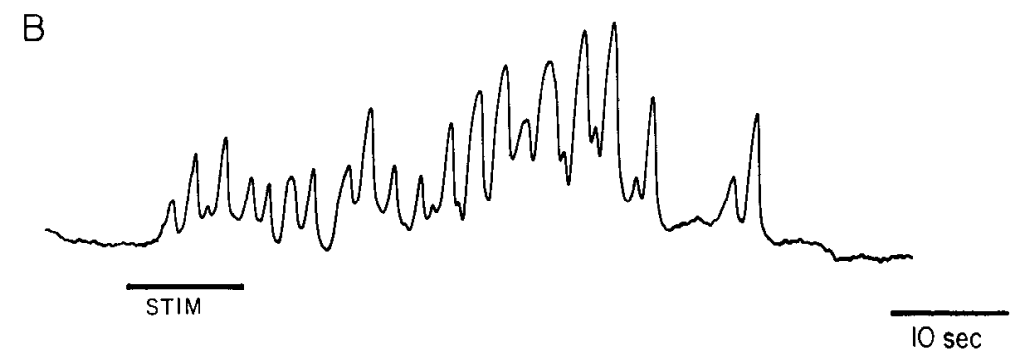




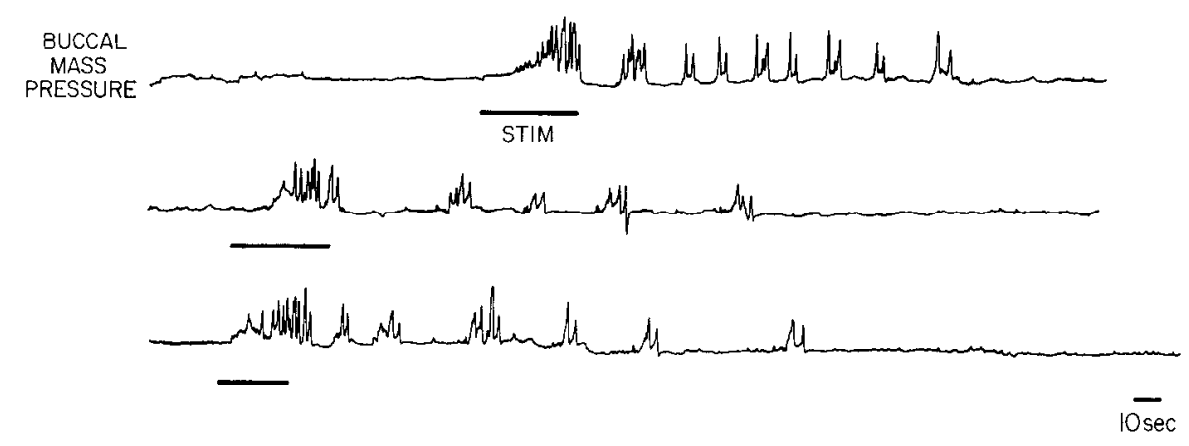

Figure 5. Prolonged effects of food stimulation in the isolated head preparation. Quiescent animals were stimulated with food (bar). This produced a bout of feeding movements. After the food was withdrawn, scveral bouts of feeding movements occurred spontaneously. As the animal became quiescent, restimulation with food caused the above pattern to repeat. delay of from $1 \mathrm{sec}$ to over $1 \mathrm{~min}$, the buccal mass moved forward and arterial blood pressure increased. Since this movement was maintained, and appeared to be a necessary concomitant to further activity, we term this phase "cocking." In very excitable preparations, cocking fuses into a single movement with the first protraction of the odontophore. Observation of the buccal mass through the body wall of intact small animals (less than $1 \mathrm{~cm}$ in length) indicated that a distinct buccal cocking phase also occurs in normal animals upon the initial presentation of food. Following cocking, a protraction-retraction sequence occurs in the isolated head preparation. If the food is then removed, several patterns of activity may follow. Further movements may cease, in which case the buccal mass "uncocks" and returns to the unstimulated state (Fig. $4 A$ ). The buccal mass may continue to show bursts of protraction-retraction movements for up to a minute before uncocking and becoming quiescent (Fig. 4B). Finally, in addition to directly evoking feeding responses, food can provoke spontaneous bouts of feeding for some time after the food stimulus has been removed (Fig. 5). Observations of free-moving intact animals revealed similar patterns of either single radula movements or trains of movements following brief exposure to food.

Buccal movements in the isolated head preparation could be elicited most reliably when the food was applied to the perioral zone or to tissue between the jaws. Stimulation in regions several millimeters from the perioral zone was often not effective. When food was maintained in contact with the perioral zone, a series of buccal movements could be evoked. In 5 preparations in which we performed measurements, the movements were regular and occurred at a ratc of $9.3 / \mathrm{min} \pm 0.81$ SEM. Each protraction movement was generally associated with a mouth opening (see small circles in Fig. $11 B$ ), but the opening was never large and sometimes was difficult to observe. The radula was never observed to rotate past the jaws.

Elsewhere (Chiel et al., 1986), we report that when rhythmic buccal movements were evoked by electrical stimulation of an esophageal nerve, food that had been placed between the jaws and in the buccal cavity moved out of the cavity, which suggests that movements induced by electrical stimulation may represent egestion rather than ingestion. All other observations of evoked buccal movements in this report used seaweed stimuli applied to the perioral zone. In vigorous isolated head preparations, perioral zone stimulation always resulted in the inward movement of seaweed placed between the jaws and the ingestion of the seaweed; that is, the seaweed moved into the buccal cavity and came out at the cut end of the esophagus.

Although our observations suggest that buccal movements in the isolated head preparation are related to ingestion rather than egestion, it is not clear whether the movements represent biting, swallowing, or a combination of these responses. Elsewhere (Chiel et al., 1986), we present evidence that buccal extrinsic muscle E4 exhibits electrical activity during swallowing or during a biteswallow, but is electrically inactive during a pure bite. We therefore recorded the electrical activity of muscle E4 in the isolated head preparations, while buccal movements were elicited by seaweed. Muscle activity was recorded by means of a modified suction electrode, using the methodology described in detail by Chiel et al. (1986). We found that buccal movements in the isolated head preparation were associated with phasic electrical activity in muscle E4 (Fig. 6; compare with Figs. 22-24 in Chiel et al., 1986).

\section{Buccal mass movements in a "feeding" isolated head preparation: detailed description}

In free-moving animals, feeding movements are typically described in terms of protraction and retraction movements of the odontophore and the attached radula. The radula of the isolated head preparation also exhibited protraction and retraction, but our observations have indicated that the movements are far more complex. Each cycle of phasic buccal movement consists of at least 3 distinct, but coordinated, movements, each of which, in turn, consists of 2 main phases. First, the whole buccal mass moves forward and backward. Second, the odontophore and radula rotate forward, toward the mouth, and backward, toward the esophagus. Finally, the radula halves open and close. Each cycle of buccal movement is associated with 2 surges of arterial blood pressure, presumably because of 2 corresponding increases of vascular resistance. Observation of the buccal mass, as well as mechanical recordings of the movement of the odontophore, suggests that increased vascular resistance during buccal movements results, at least in part, from active contraction of those buccal muscles that foreshorten the buccal mass (Fig. $7, B, D)$. This contraction may shrink the spongy sinus that ordinarily provides a low-resistance path for blood flow. Pressure recordings indicate that each of the forward and backward rotations of the odontophore consists of two subphases, which we will refer to as the "active phase" and the "return to rest."

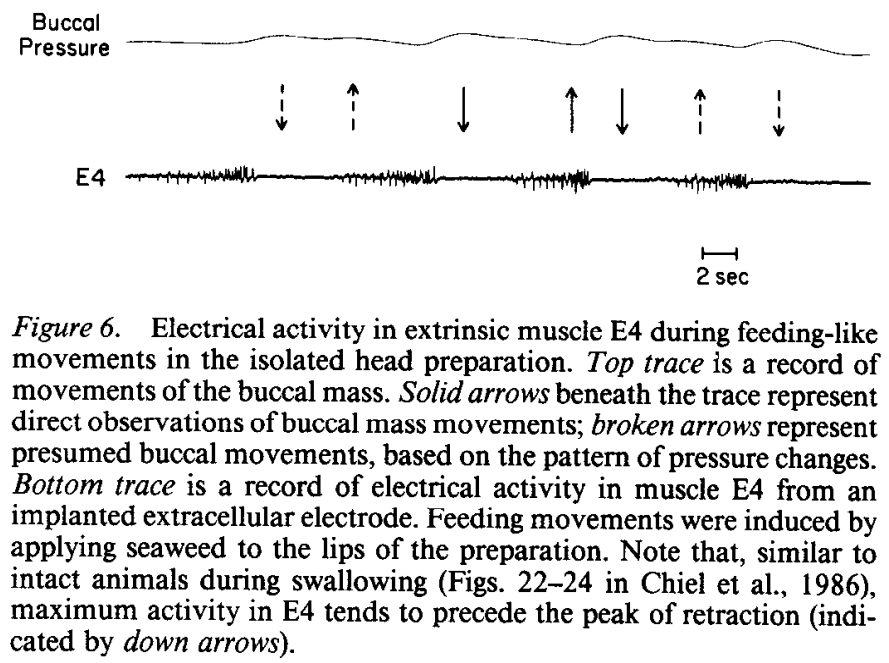




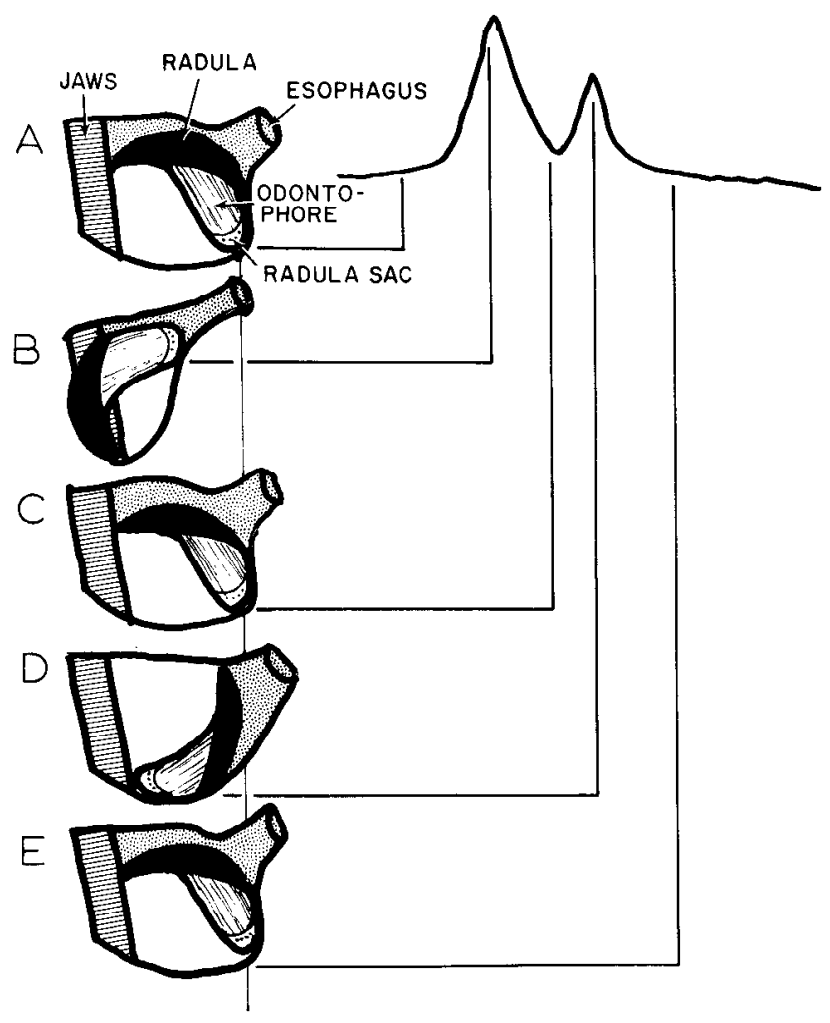

Figure 7. Diagrammatic representation of movement of the odontophore with attached radula (black) and of changes in the shape and position of the buccal mass during a single bite-swallow cycle. Idealized cross sections of the buccal mass are shown. Buccal cavity is indicated by stippling. Relative proportions of jaw sizes (horizontal stripes) and buccal mass are based on measurements of buccal masses from animals weighing $100-200 \mathrm{gm}$. Five cross sections, representing different phases of the cycle, are shown. Also shown is an idealized transducer record of movement of the tissue just superficial to the radula sac at various times through the cycle. Position of this tissue at the onset of the cycle is indicated by thin vertical lines. A recording of fluid pressure in the buccal artery shows a similar record with approximately the same phase relations as the movement transducer (see Fig. 3), although the relative magnitudes of the phases differ. $A$, Rest position. The radula is in an approximately horizontal position in the buccal cavity. $B$, Active protraction. The radula rotates forward, away from the esophagus (small oval) and towards the jaws (cross-hatching). The buccal mass simultaneously moves forward. During biting, at the peak of active protraction, the radula moves past the mouth and jaws. $C$, Return retraction. Following the peak of active protraction, the radula rotates backwards and returns to the rest position. $D$, Active retraction. The radula rotates backward toward the esophagus. The tissue over the radula sac moves forward again. $E$, Return protraction. Cycle is completed by the radula returning to the rest position.

Thus, wc obscrve that forward rotation is associated with a pressure surge (Fig. $7 B$ ), whereas return towards the baseline or rest condition is associated with a return of blood pressure towards a baseline state (Fig. $7 C$ ). Following return to a basal condition, the odontophore shows a backward rotation which again is associated with a surge of arterial pressure (Fig. $7 D$ ), followed by a return to baseline as the odontophore again returns to the baseline condition (Fig. $7 E$ ). The odontophore therefore undergoes 2 protraction movements. One begins when the odontophore is in its central, or basal, state. This movement is associated with a strong contraction of the muscles forming the spongy sinus, with a concomitant increase of arterial resistance. We refer to this movement as "active protraction" or simply "protraction." The second protraction movement begins when the odontophore is fully retracted. This movement is associated with a return of arterial blood flow toward a baseline state, and we refer to this as "return protraction." The 2 retraction movements of the odontophore are mirror images of the protraction movements, i.e., an active phase (retraction) initiated from a central position and a return phase (return retraction) that begins at the peak of forward rotation and results in a return to the central position. In addition to protraction and retraction movements, there is, throughout a feeding bout, tonic contraction of the buccal mass, associated with tonic elevation of blood pressure, as observed in lengthy recordings of blood pressure during feeding in free-moving animals (Koch and Koester, 1982).

\section{Plasticity of pattern of buccal mass movements}

Our observations of the radula movement in the isolated head preparation suggested the hypothesis that backward movement was not simply the cessation of forward movement, and that separate motor programs may control movement of the radula forward from rest (and the return to rest) and backward from rest (and the return to rest). To explore this idea we examined whether the magnitude and phase relations of these movements were fixed or showed variation. Our data indicate that both magnitude and phase are variable, supporting the suggestion that radula movement forward from rest position and the movement backward from rest position may be driven by separate oscillators. During the course of a single bout of repetitive buccal movements, the ratio of the magnitude of the forward from rest movement to that of the backward from rest movement could change markedly (e.g., Figs. 3, 11 $A_{2}$ ). In addition to changes in the relative magnitudes of backward and forward movements, the time between the peaks of the forward and backward movements is variable.

\section{Activity of $C 2$ and the $M C C$ in the isolated head preparation}

In preparations of isolated ganglia (see Fig. 14 in Chiel et al., 1986), neuron C2 was never observed to exhibit significant excitatory synaptic input. We therefore studied its activity in the isolated head preparation. In this preparation, C2 never exhibited spontaneous activity in the absence of movement of the buccal mass or sensory stimulation of the perioral region. When a single feeding movement was elicited by brief stimulation of the perioral zone with seaweed, $\mathrm{C} 2$ fired each time the buccal mass moved (Fig. 8). Under conditions where single responses were elicited, the exact phase of firing of $\mathrm{C} 2$ relative to specific phases of buccal movement was variable, sometimes occurring late during the movement, at retraction (Fig. $8 A_{1}$ ), and sometimes occurring at the onset of the movement, during cocking (Fig. $8 A_{2}$ ).

Firing of $\mathrm{C} 2$ was accompanied by the appearance of small fast prepotentials, which decreased in size when $C 2$ was hyperpolarized (Figs. $8 B, 10 B$ ). These observations suggested that $\mathrm{C} 2$ may be an afferent neuron and that the fast prepotentials may be blocked axon spikes conducted from the periphery towards the soma. This conclusion is supported by data presented in a following paper (Weiss et al., 1986b).

Seaweed provides both chemical and mechanosensory stim$u l i$. To determine if activation of $\mathrm{C} 2$ was exclusively dependent upon the chemical stimulation provided by the seaweed, we tested several mechanical stimuli. We found that even in the absence of feeding-like responses of the buccal mass, tonic firing of $\mathrm{C} 2$ could be elicited by stimulating the perioral zone of the preparation with filter paper (Fig. $9 A$ ) or by spreading the jaws, either with forceps or by inflating a balloon inserted into the mouth. When the mouth of the animal was opened with forceps, firing of $\mathrm{C} 2$ was accompanied by fast prepotentials (Fig. 10A) which decreased in size as C2 was hyperpolarized (Fig. 10B).

Since C2 appears to fire in response to mechanostimuli associated with the perioral zone, its firing in response to a chemostimulus applied to the mouth may consist of a direct re- 


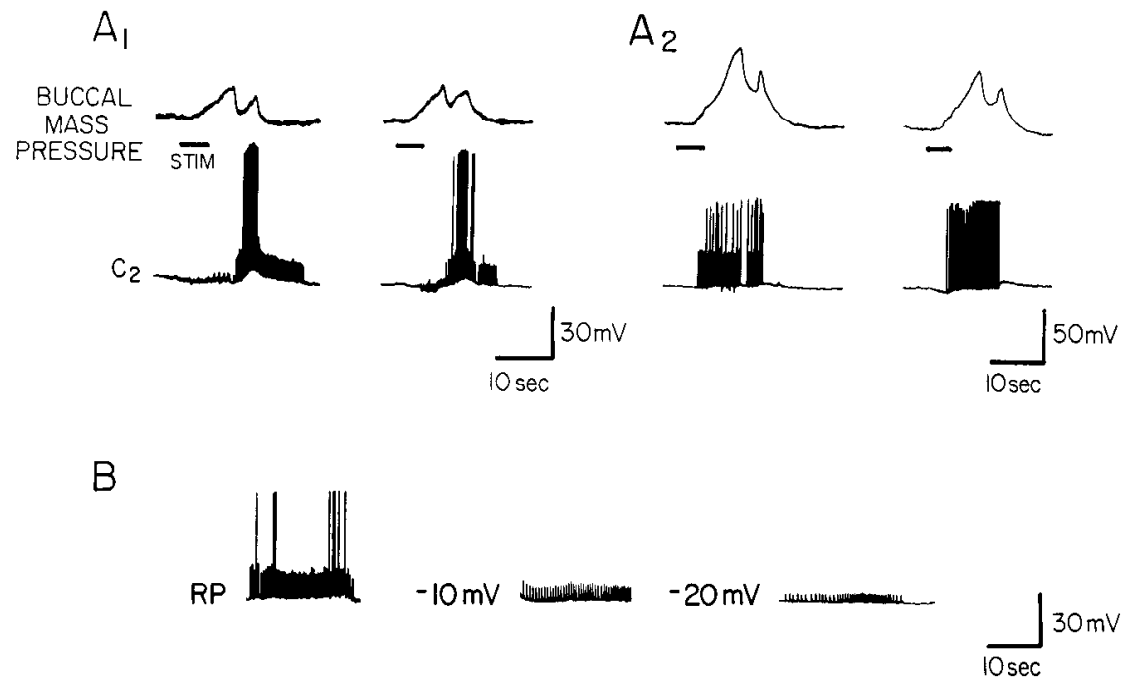

Figure 8. Activity of $\mathrm{C} 2$ during individual feeding responses. $A$, Two records from individual animals $\left(A_{l}\right.$, $A_{2}$ ). These records indicate that when single responses were elicited, $\mathrm{C} 2$ fired. Activity of $\mathrm{C} 2$ was accompanied by fast depolarizing potentials. $B$, When $\mathrm{C} 2$ was hyperpolarized relative to resting potential $(R P)$, the fast depolarizing potentials decreased in magnitude, suggesting that they may be blocked axon spikes (A-spikes). sponse to the tactile properties of the stimulus, as well as an indirect response associated with movements of the buccal mass during cocking and protraction or retraction movements. To examine the relationship of buccal movement to firing of $\mathrm{C} 2$, we examined feeding bouts of long duration. Figure $11 A$ shows the firing pattern of $\mathrm{C} 2$ in 2 different animals during stimulation by seaweed, which induced prolonged bouts of forward-backward movements of the buccal mass. $\mathrm{C} 2$ fired once during each double cycle. The firing began during return retraction or at the onset of active retraction, and appeared to be linked to the time of mouth closing (Fig. $11 B$ ). Firing continued during the phase of active retraction. Termination of firing was typically associated with a burst of IPSPs in the cell, followed by axon spikes (A-spikes) and then total cessation of activity in $\mathrm{C} 2$. Spike activity of $\mathrm{C} 2$ often terminated at the end of return protraction, whereas A-spikes could continue until the next bout of active protraction. To determine whether continued stimulation with seaweed was needed to produce activity of $\mathrm{C} 2$, feeding-like movements were elicited by seaweed, which was then removed. In many instances, thythmic movements of the buccal mass persisted, and in these cases $\mathrm{C} 2$ maintained its activity in phase with the movements (Figs. 11B, 12). In some instances, during a low rate of spontaneous buccal movements, the buccal mass underwent a spontaneous cocking phase and an increased rate of buccal movements was indicated (Fig. $11 B$ ). C2 showed a corresponding increase in the rate of its spike-bursting.

\section{Contribution of $\mathrm{C2}$ to activity of the $\mathrm{MCC}$}

Since C2 provides excitatory input to the MCC, we wished to examine the activity recorded from the 2 cells simultaneously in the isolated head preparation (Fig. 12). The spike activities
$A$

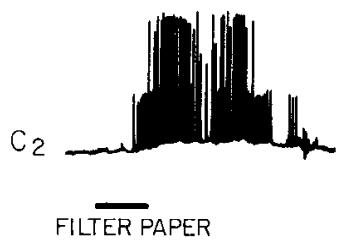

$B$

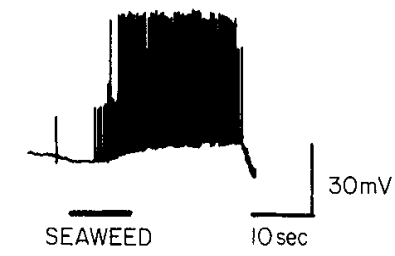

Figure 9. Activation of neuron $\mathrm{C} 2$ by seaweed $(B)$ and glass fiber filter paper $(A)$. A purely mechanical stimulus can evoke a substantial response in $\mathrm{C} 2$. of $\mathrm{C} 2$ and the MCC were roughly parallel, although $\mathrm{C} 2$ fired more readily and at a higher frequency than the $\mathrm{MCC}$.

To determine whether firing of $\mathrm{C} 2$ contributed to the spike activity of the MCC during stimulation of the animal with food, MCC firing was determined in successive runs of food stimulation, with and without firing of the ipsilateral $\mathrm{C} 2$. When $\mathrm{C} 2$ was prevented from firing by means of continuous hyperpolarizing currents, the MCC showed weaker activity than in the preceding or following run, when $\mathrm{C} 2$ was not hyperpolarized and fired normally. Although hyperpolarization of $\mathrm{C} 2$ could apparently completely block activity of the MCC (Fig. 13), in most runs removing $\mathrm{C} 2$ reduced but did not eliminate activity of the MCC (mean reduction, $40 \%$ in 4 preparations). This suggests that cells other than $\mathrm{C} 2$ also contribute to the excitatory input to the MCC during stimulation with food and during driving of the feeding motor program. Studies by Ono and McCaman (1980) indicate that C2 is electrically coupled to another cell in the E cluster. This cell is also histaminergic, and exerts many of the synaptic effects that $\mathrm{C} 2$ does. It is possible, therefore, that one source of input to the MCC when $\mathrm{C} 2$ is

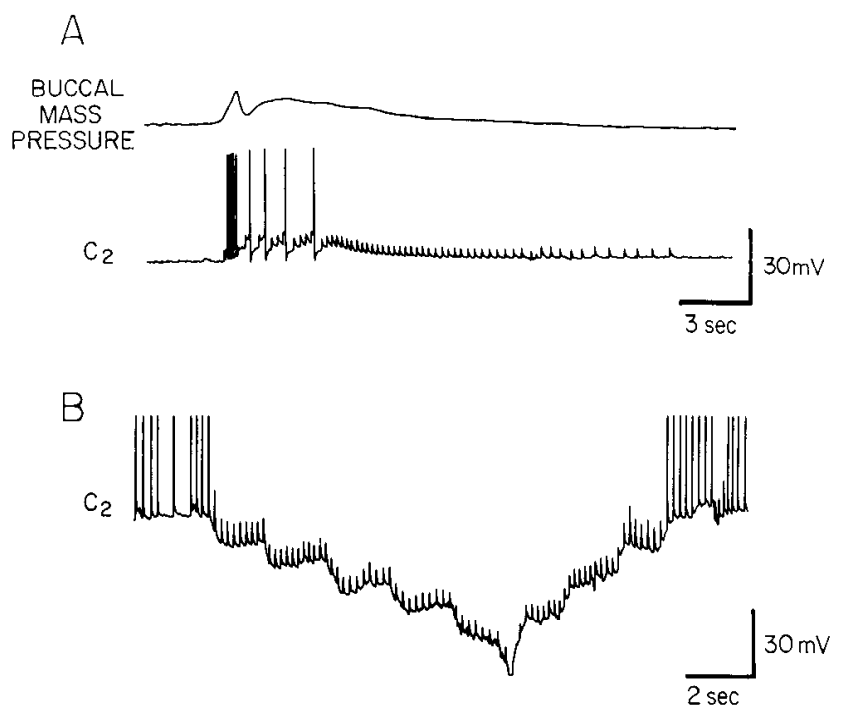

Figure 10. A, Spreading the jaws of the animal with forceps can activate firing of $\mathrm{C} 2$ and bring in fast depolarizing potentials. $B$, As in the case for responses elicited by food stimuli (Fig. 8), hyperpolarization of $\mathrm{C} 2$ reduces the size of the fast potentials. 

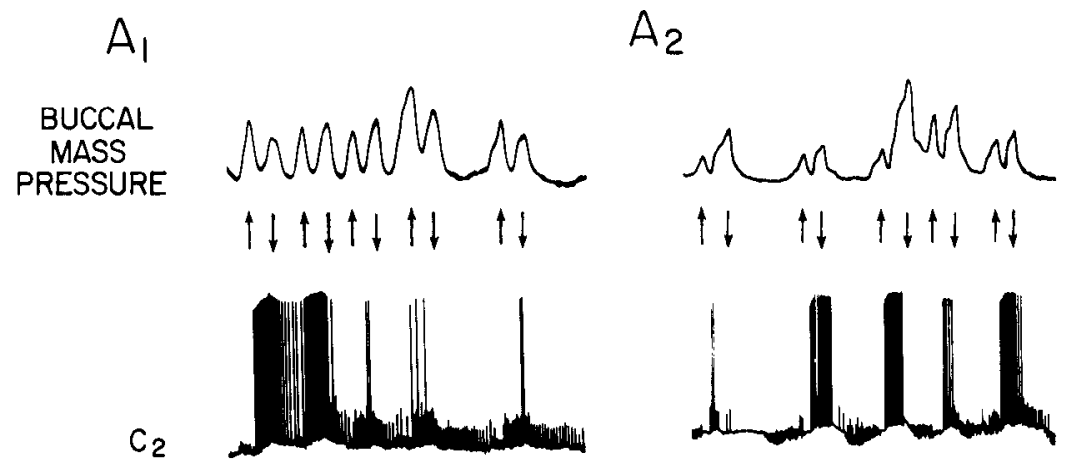

Figure 11. Activity of $\mathrm{C} 2$ during bouts of feeding responses. $A$, Records from 2 different animals exhibiting a similar pattern of activity of C2. $A_{1}$ and $A_{2}$, Animals were stimulated with seaweed throughout the feeding bout. $B$, A record of feeding and $\mathrm{C} 2$ activity after the seaweed has been removed. The responses thus recorded are not contaminated by the stimulating characteristics of the seaweed. In addition to monitoring radula movements, we indicated the time of mouth closure of the animal (small circles), which in this case could be monitored unobstructed by the seaweed. Arrows indicate peak of forward $(u p)$ and backward (down) movements of the buccal mass, as described in Figure 3.

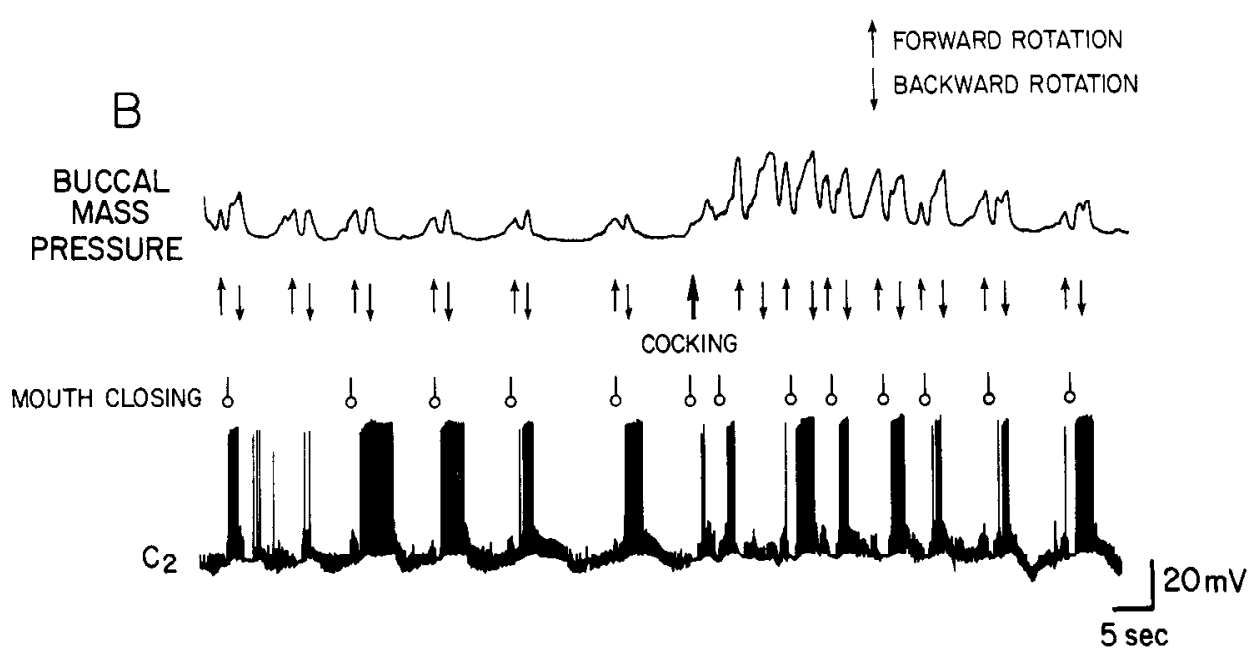

hyperpolarized is this other electrically coupled, histaminergic neuron. This hypothesis is consistent with the observation illustrated in Figure 13, where strong hyperpolarization of cell C2 can completely block the excitatory input to the MCC.

Contribution of $M C C$ activity to buccal mass movements in the isolated head preparation

In previous studics of the isolated buccal ganglion, we demonstrated that activity of the MCC increases the frequency of the feeding motor program (Weiss et al., 1978). The development of the isolated head preparation permitted us to determine whether the MCC exerted similar effects on feeding-like movements. In 3 preparations, multiple trials of stimulation with seaweed were run, while the activity of the MCC was promoted or inhibited by means of intracellular currents. In one experiment, we succeeded in controlling both MCCs. When the MCCs were hyperpolarized so that they did not exhibit any spikes during seaweed stimulation of the lips, the buccal mass exhibited weak movements, which occurred at a slow rate (Fig. 14, $A, C$ ). When both MCCs were fired, the buccal mass movements were greatly enhanced (Fig. 14B).

\section{Discussion}

The present research is part of our attempt to define the neurons that might comprise elements of a central arousal system that elicits and maintains the food arousal state in Aplysia. In the research reported in the previous paper (Weiss et al., 1986a), we looked for neurons that excite the serotonergic MCC, a neuron that mediates several of the behavioral manifestations of behavioral arousal elicited by food. We found that the histaminergic neuron $\mathrm{C} 2$ produces a slow excitatory input to the $\mathrm{MCC}$. In order to study the possible behavioral role of $\mathrm{C} 2$, we developed an improved semi-intact preparation which exhibits feeding-like behavior. Using this preparation, we have now found that (1) feeding movements of the buccal mass consist of several distinct components that may be independently controlled; (2) neuron $\mathrm{C} 2$, although not active when the isolated nervous system executes a central program, shows spike activity that is phasically locked to feeding-like behavior in the isolated head preparation; this suggests that $\mathrm{C} 2$ may be driven by afferent activity rather than by a central program; and (3) the activity of $\mathrm{C} 2$ contributes to the firing of the MCC during feeding behavior and, in turn, the activity of the MCC contributes to feeding-like behavior in the isolated head preparation.

\section{The nature of buccal mass movements in the} isolated head preparation

One problem in the analysis of feeding in Aplysia has been the ease with which feeding behavior is suppressed in this animal. In particular, noxious stimuli establish a defensive arousal state and suppress feeding for a much longer time (Kupfermann and Pinsker, 1968) than appears to be the case for several other mollusks that have been successfully used in semi-intact preparations (Croll et al., 1985a, b; Gillette and Gillette, 1983; Reingold and Gelperin, 1980; Rose and Benjamin, 1979; Susswein et al., 1984a). The great sensitivity of Aplysia to its internal state provides an interesting parallel to the operation of motivational states in higher animals, but it also makes it difficult to study feeding in semi-intact preparations. These preparations can be set up only by inflicting extensive trauma on the animal, and invariably function under less than optimal physiological conditions.

The isolated head preparation we have developed shows more vigorous and sustained activity than previous semi-intact prep- 

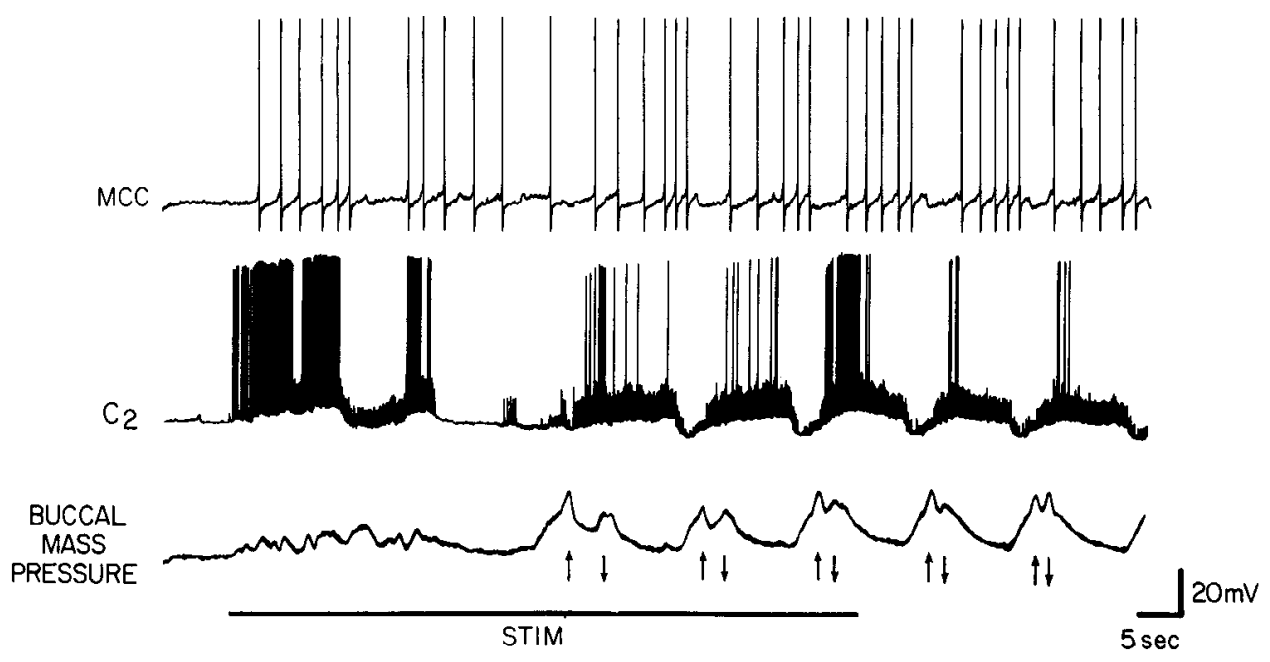

Figure 12. Simultaneous activity of $\mathrm{C} 2$ and the MCC during feeding initiated by seaweed applied to the perioral zone and mouth (STIM). Forward and backward movements of the buccal mass are indicated by the arrows, as described in Figure 3. arations capable of buccal movement in Aplysia (Koch et al., 1984). Behavioral observations of Aplysia suggest that the buccal mass mediates ingestive behaviors as well as an egestive behavior (rejection). We cannot exclude the possibility that there may be other buccal mass behaviors that we have not discovered, but several observations strongly favor the idea that our isolated head preparation exhibits a form of food-ingestive behavior. Protraction-retraction movements are specifically elicited by food stimuli applied to the perioral zone. Similar to normal feeding, buccal movement begins with a cocking phase, followed by movements occurring with a regular rhythm. Furthermore, the movements are organized into patterns that are reproducible and recognizable from preparation to preparation as well as between runs in a given preparation. Finally, the movements can result in food being drawn into the buccal cavity and the esophagus.

The current observations on intact animals support the previous conclusion (Kupfermann, 1974b) that there are 2 types of ingestive movements: biting and swallowing. We cannot at present distinguish between these 2 types of movement in the isolated head preparation, but several observations suggest that we may be eliciting predominantly swallowing, or a weak bite followed by a swallow (bite-swallow), rather than pure biting. First, food stimuli were effective only when presented to the perioral region or between the jaws, and we cannot exclude the possibility that dissolved phagostimulants entered the buccal cavity. In the intact animal, biting movements are most effectively elicited by stimulation of the perioral zone, but can also be elicited by stimulation of an area several millimeters beyond that zone. Second, unlike biting but similar to swallowing, the mouth never opened wide in the isolated head preparation, and the radula did not protract beyond the jaws. Finally, we have evidence (Chiel et al., 1986) that feeding movements in the isolated head preparation are associated with muscle activity of the extrinsic buccal muscle E4. This observation suggests that buccal movements in the isolated head preparation are not biting movements, since, in intact animals, this muscle shows little or no activity during biting, but is active during swallowing, bite-swallows, and rejection. Nevertheless, conditions in the isolated head preparation are sufficiently different from those in the intact animal to make direct comparison subject to error. Thus, for example, the restricted receptive field and lack of full mouth opening in the isolated head preparation might not be an indication that the movements represent swallowing but, rather, may be due to the fact that this preparation is less responsive than the intact animal. Weak biting responses occur in normal animals during low arousal, as animals satiate (Susswein et al., 1976).

\section{Independent controls of components of buccal movements}

In an initial observation of intact animals, the consummatory phase of feeding appeared to consist of simple protraction-re-
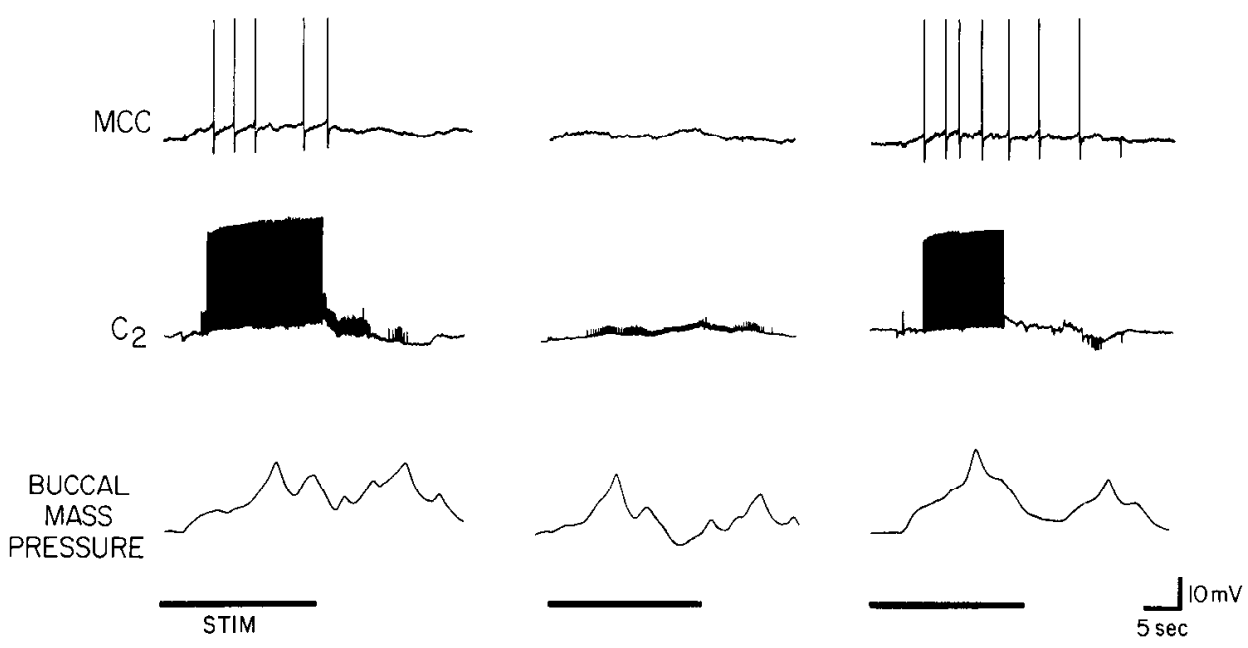

Figure 13. Effect of hyperpolarizing neuron $\mathrm{C} 2$ on the firing of the MCC in response to stimulation with seaweed. Top, $\mathrm{C} 2$ was not hyperpolarized, and its firing was accompanied by the firing of the MCC. Middle, Hyperpolarization of $\mathrm{C} 2$ prevented the firing of the MCC. Bottom, Hyperpolarization was removed and firing of $\mathrm{C} 2$ in response to seaweed again led to firing of the MCC. 
A
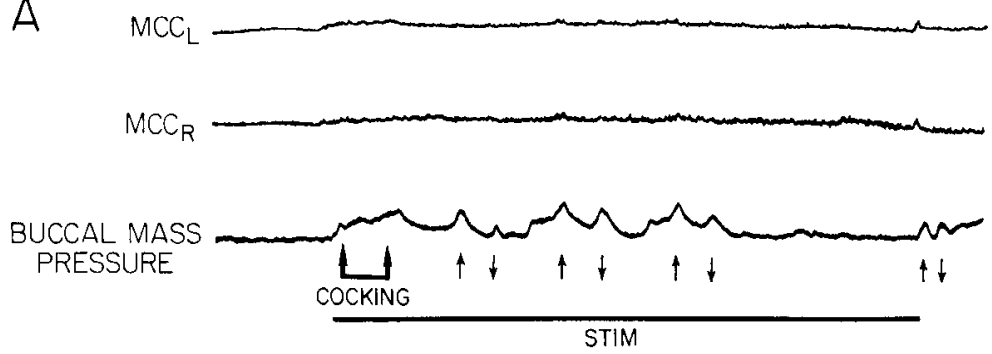

B
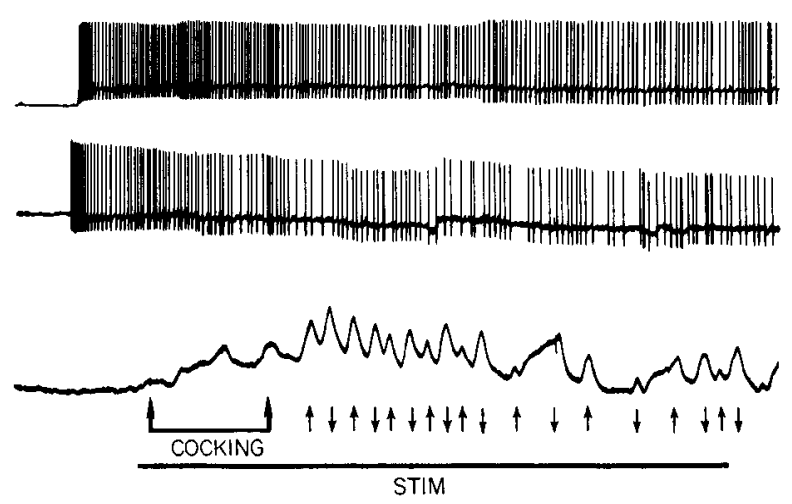

C

Figure 14. Effect of MCC activity on food-evoked feeding behavior. $A$ and $C$, Left and right metacerebral cells $\left(M C C_{L}\right.$ and $\left.M C C_{R}\right)$ were hyperpolarized while the animal was stimulated with seaweed. The resulting feeding activity was less than when the MCCs were depolarized and fired in $B$.

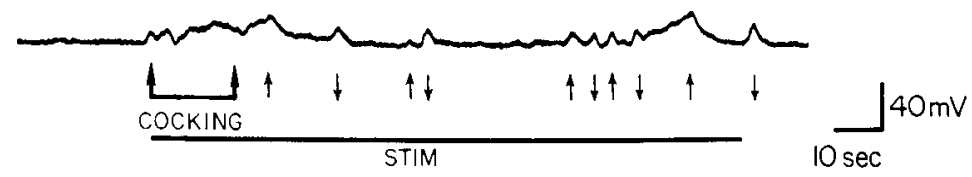

traction movements of the radula, coordinated with opening and closing of the jaws and surrounding structures. Further observations revealed that, in addition, feeding involved opening and closing of the 2 halves of the radula. Our observations of the isolated head preparation have indicated that the protraction-retraction movements consist of at least 2 subphases of radula movement: (1) forward rotation and return to rest, and (2) backward rotation and return to rest. In some instances, the 2 subphases blend into one another, so that an uninterrupted forward and backward movement results. In other instances, the forward and backward movements are separated in time and can be clearly differentiated.

Our studies suggest that the 2 subphases of the motor program are linked but are under separate neural controls. These 2 behaviors appear in sequence, but their relative intensity, duration, and phase relations are variable. Such "plasticity" in the elements of the motor program can have 2 important consequences: (1) The same set of muscles can execute different behaviors, depending on the motor program that impinges upon them. Thus strong forward movements of the radula may be necessary to guarantee a strong purchase on food when an animal bites; in contrast, strong backward movements of the radula may be necessary for swallowing. (2) The program can deal effectively with variation in the properties of the food the animal ingests. Thick, chunky pieces of seaweed may require more vigorous biting and swallowing than do thin pieces.

In addition to forward and backward rotation of the radula, the semi-intact preparation exhibited a cocking phase involving tonic protraction of the whole buccal mass. In fact, forward and backward movement always occurred against a background of tonic protraction of the buccal mass. Cocking may be analogous to the preparatory phase that precedes other invertebrate rhythmic behaviors, e.g., swimming in the leech (Kristan and Weeks, 1983) and jumping in locusts (Pearson, 1982). Cocking is also a feature of rhythmic behaviors in mammals. For example, in scratching behavior in the cat, flexion and extension leg movements always occur against a background of leg flexion and are suppressed if the leg is manually extended (Berkinblit et al., 1978). The behavioral similarities of buccal movements and scratching in vertebrates invite the speculation that some aspects of the neuronal model suggested for scratching in the cat may be applicable to feeding in Aplysia (see also Croll et al., 1985b).

Figure 15 presents a hypothetical model that, at this stage of empirical evidence, is unlikely to be correct in detail but that nevertheless summarizes the element we feel are likely to comprise the motor system controlling buccal movements in Aplysia. Unlike scratching in the cat, which may be driven by a pair 
of generators of flexion and extension, buccal mass movements appear to require at least 2 pairs of generators: one associated with rotation of the radula forward from rest, and a second associated with rotation of the radula backward from rest. These primary generators, which are presumably driven by command systems for swallowing or biting, drive at least 3 pairs of motor neuron pools, which control 3 types of movement: buccal mass protraction/retraction, odontophore forward/backward rotation, and radula opening/closing. These motor neuron pools may share some common elements and are represented in Figure 15 by 3 overlapping circles. The isolated buccal ganglion is capable of the rhythmic and organized bursting of buccal motor neurons (Gardner, 1977; Weiss et al., 1978), suggesting that the generators of buccal movement may function without peripheral afferent control. Buccal movements, however, appear to be modulated by a variety of sources of peripheral feedback (JahanParwar et al., 1983; Kupfermann and Cohen, 1971; Laverack, 1970; Rosen et al., 1982). In our model, similar to that proposed by Berkinblit et al. (1978), we postulate that movement of the buccal mass, specifically tonic forward movement, or cocking, provides feedback to the pattern generator, which aids cycling. Finally, we have added a pathway from the egestion command to the buccal mass motor neurons, which can function to produce the initial cocking response that occurs before any rhythmic movements.

\section{Activity of $C 2$ during feeding}

Our evidence indicates that identified histaminergic neuron $\mathrm{C} 2$ provides slow excitatory synaptic input to the MCC and that $\mathrm{C} 2$ is phasically active during buccal mass movements in the isolated head preparation. We have found that $\mathrm{C} 2$ is not active during the generation of motor programs when the CNS is disconnected from the periphery. It is not certain that the motor programs in isolated ganglia are identical to those generated when peripheral structures are present. Nevertheless, the absence of $\mathrm{C} 2$ spike activity in the isolated nervous system suggests that this neuron may be driven by afferent activity associated with feeding motor movements. Indeed, in a companion paper (Weiss ct al., 1986b), we show that C2 itsclf is an afferent neuron that responds to mechanical stimuli emanating from the perioral region of the animal, even when chemical synaptic transmission is blocked. Thus, part of the response of $\mathrm{C} 2$ in the isolated head preparation, particularly at the onset of feeding, may be directly elicited by tactile stimulation provided by the seaweed when it is pressed to the lips of the animal. But once rhythmic buccal movements occur, C2 activity persists even when food is removed. Each backward movement of the buccal mass and concomitant closing of the jaws is associated with spike activity in C2 due, at least in part, to activation of the peripheral axon of $\mathrm{C} 2$. In other words, rather than being activated by a central program, $\mathrm{C} 2$ is activated by some aspect of feeding behavior itself, perhaps jaw closure.

\section{Role of $C 2$ in activating the metacerebral cell and maintaining arousal}

One of the features of feeding behavior in Aplysia is that, once it is initiated, the behavior and the food arousal state persist for a period of time. This type of "behavioral inertia" is also characteristic of behavior in vertebrates, and it has been postulated that behavioral inertia can be explained by positive feedback mechanisms (Golani et al., 1979; Roberts et al., 1984; Wiepkema, 1971). When Aplysia are briefly exposed to food, the MCC becomes active and remains active for up to a minute or two after the food stimulus is removed (Kupfermann and Weiss, 1982). Reactivation of the MCC and latency to respond to subsequent food are facilitated for up to $1 / 2 \mathrm{hr}$ after the initial presentation of food (Kupfermann and Weiss, 1982; Susswein et al., 1978). Previous evidence, together with the current exper-

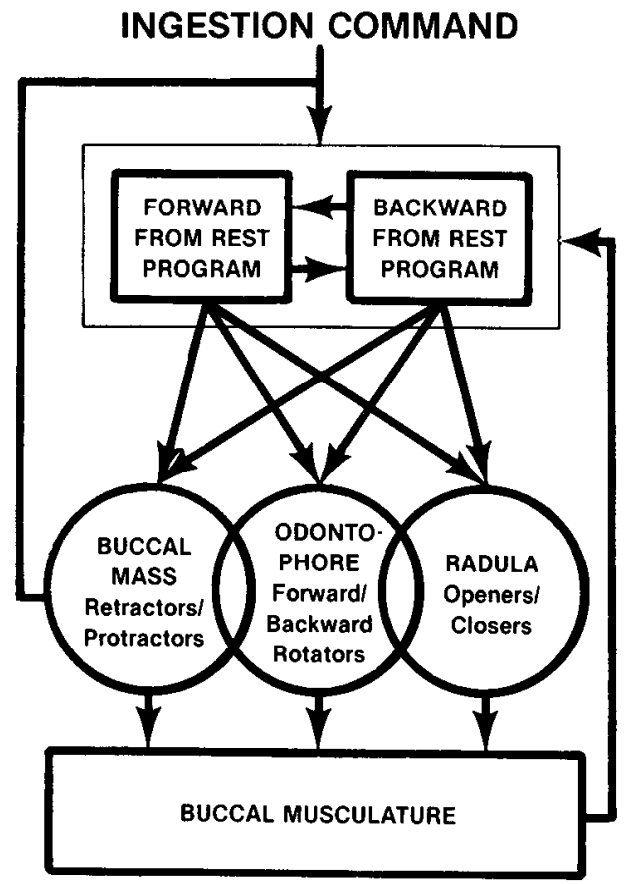

Figure 15. Hypothetical model of the neural organization of systems controlling the buccal musculature. A feeding command signal activates a dual pattern generating system, which in turn drives 3 sets of motor neurons involved in producing 3 types of movement. Feedback from afferents activated by movement or muscle tension is indicated by the arrow from the muscle.

iments, indicate that the activity of the MCC facilitates both the magnitude and frequency of feeding responses involving the buccal mass (Weiss et al., 1982). Our current observations suggest that at least part of the reason the MCC maintains activity when the initiating food stimulus is no longer present is that the behavior elicited by the food stimulus evokes afferent activity in $\mathrm{C} 2$, a neuron that excites the MCC. Parallel excitation of the MCC may be provided by cells such as C3 (Ono and McCaman, 1980), which are electrically coupled to C2 and have similar synaptic outputs. Even though firing of $\mathrm{C} 2$ is phasic, it produces very slow synaptic potentials in the MCC and, therefore, it should exert relatively weak phasic effects upon firing of the MCC. In turn, the effects of a burst of spikes of the MCC on muscle and central circuits extend over many cycles of feeding movements. Therefore, the phasic firing of $\mathrm{C} 2$ should result primarily in a tonic arousal effect on biting. Our evidence suggests that $\mathrm{C} 2$ and the MCC may be part of a positive feedback loop in which activity of the MCC promotes buccal mass movements, which trigger activity of $\mathrm{C} 2$. $\mathrm{C} 2$ in turn excites the MCC, which further promotes buccal movements. Neuron C2 may therefore contribute to the persistence of feeding behavior once feeding is initiated. The positive feedback loop that appears to be activated during feeding is insufficient to maintain feeding behavior in the absence of food since, when food is removed, biting responses generally cease after a few minutes and the MCC becomes silent. Nevertheless, activity of $\mathrm{C} 2$ appears to be one of many factors that promote continuity of behavior by maintaining arousal and behavioral responsiveness even when the eliciting stimuli are no longer present. Although this hypothesis is supported by the existing data, it remains speculative since we have not directly tested the effects of removal of $\mathrm{C} 2$ on arousal. This experiment, although possible in principle, is difficult to perform with our current isolated head preparation since this preparation does not exhibit entirely normal biting behavior 


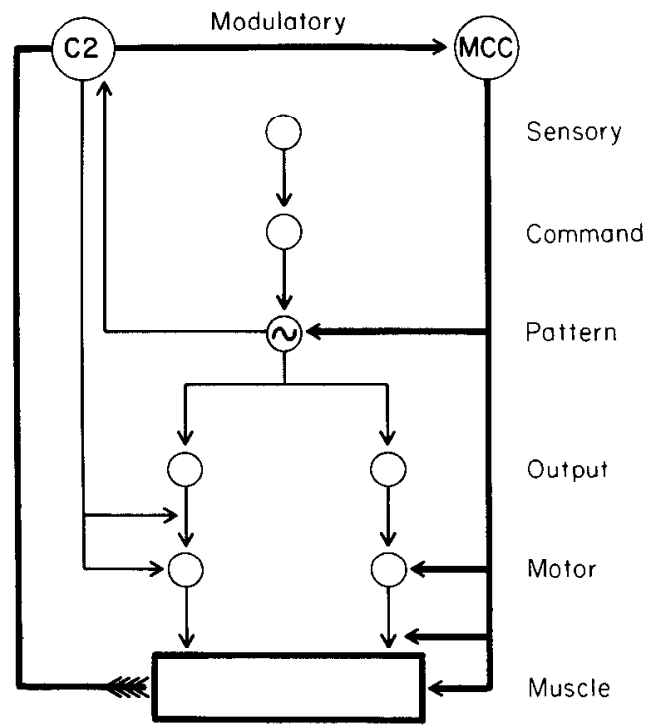

Figure 16. Schematic diagram of hypothetical levels of control of neurons controlling consummatory feeding responses in Aplysia. Types of neurons for which there is evidence in Aplysia and other gastropod mollusks include the following: sensory cells or sensory neurons, which excite command neurons or systems of command neurons. Command neurons drive central pattern generators, whose output to motor neurons either can be direct or, as indicated, can be mediated by way of output neurons. Various levels of control are influenced by at least 2 types of modulatory neurons: $\mathrm{C} 2$ and the MCC. Feedback loop involving C2 and the MCC is indicated by dark lines.

and does not show the vigorous arousal response seen in intact animals (Kupfermann and Weiss, 1982).

In the following papers of this series (Chiel et al., 1986; Weiss et al., 1986b), we provide evidence that $\mathrm{C} 2$ is a mechanosensory cell and that it is synaptically interconnected with other cells that are involved in aspects of feeding behavior other than the arousal functions mediated by the MCC. Figure 16 is a schematic summary of the circuits controlling consummatory feeding responses in Aplysia and illustrates (dark lines) the positive feedback loop discussed in this and the preceding paper. It is not known whether positive feedback exists at levels other than the mechanoafferents, but in Pleurobranchaea (Gillette et al., 1978) and Lymnaea (McCrohan and Benjamin, 1980), feedback is found at the level of motor or command elements for feeding.

The results reported in the present paper are consistent with the idea that $\mathrm{C} 2$ can be considered part of an arousal system that has been hypothesized as maintaining the food arousal state in Aplysia (Kupfermann and Weiss, 1981; Weiss et al., 1982). The data in the following papers (Chiel et al., 1986; Weiss et al., 1986b), however, indicate that the other properties of $\mathrm{C} 2$ do not readily fit the idea of $\mathrm{C} 2$ as being an element of a central arousal system that has very broad effects, but suggest, rather, that $\mathrm{C} 2$ is one of a number of neurons that have relatively restricted modulatory effects.

\section{References}

Berkinblit, M. B., T. G. Deliagina, A. G. Feldman, I. M. Gelfand, and G. N. Orlovsky (1978) Generation of scratching. II. Nonregular regimes of generation. J. Neurophysiol. 41: 1058-1069.

Carew, T. J., and E. R. Kandel (1977) Inking in Aplysia californica. I. Neural circuit of an all-or-none behavioral response. J. Neurophysiol. 40: 692-707.

Chiel, H. J., K. R. Weiss, and I. Kupfermann (1986) An identified histaminergic neuron modulates feeding motor circuitry in Aplysia. J. Neurosci. 6: 2427-2450.

Croll, R. P., W. J. Davis, and M. P. Kovac (1985a) Neural mechanisms of motor program switching in the mollusc, Pleurobranchaea: I. Central motor programs underlying ingestion, egestion and the "neutral" rhythm(s). J. Neurosci. 5: 48-55.

Croll, R. P., M. P. Kovac, W. J. Davis, and E. M. Matera (1985b) Neural mechanisms of motor program switching in the mollusc, Pleurobranchaea. III. Role of the paracerebral neurons and other identified brain neurons. J. Neurosci. 5: 64-71.

Gardner, D. (1977) Interconnections of identified multiaction interneurons in buccal ganglia of Aplysia. J. Neurophysiol. 40: 349-361.

Gillette, M. U., and R. Gillette (1983) Bursting neurons command consummatory feeding behavior and coordinated visceral receptivity in the predatory mollusk Pleurobranchaea. J. Neurosci. 3: 1791-1806.

Gillette, R., M. P. Kovac, and W. J. Davis (1978) Command neurons in Pleurobranchaea receive synaptic feedback from the motor network they excite. Science 199: 798-801.

Golani, I., D. L. Wolgin, and P. Teitelbaum (1979) A proposed natural geometry of recovery from akinesia in the lateral hypothalamic rat. Brain Res. 164: 237-267.

Hening, W. A., E. T. Walters, T. J. Carew, and E. R. Kandel (1979) Motoneuronal control of locomotion in Aplysia. Brain Res. 179: 231253.

Jahan-Parwar, B., and S. M. Fredman (1978) Control of pedal and parapodial movements in Aplysia. I. Proprioceptive and tactile reflexes. J. Neurophysiol. 41: 600-608.

Jahan-Parwar, B., A. H. Wilson, Jr., and S. M. Fredman (1983) Role of proprioceptive reflexes in control of feeding muscles of Aplysia. J. Neurophysiol. 49: 1469-1480.

Koch, U. T., and J. Koester (1982) Time-sharing of heart power: Cardiovascular adaptations to food arousal in Aplysia. J. Comp. Physiol. 149: 31-42.

Koch, U. T., J. Koester, and K. R. Weiss (1984) Neuronal mediation of cardiovascular effects of food arousal in Aplysia. J. Neurophysiol. 51: 126-135.

Kristan, W. B., Jr., and J. C. Weeks (1983) Neurons controlling the initiation, generation and modulation of lccch swimming. In Neural Origin of Rhythmic Movements, A. Roberts and B. Roberts, eds., pp. 243-260, Society of Experimental Biology, Symposium No. 37, The Company of Biologists Ltd., Cambridge, UK.

Kupfermann, I. (1974a) Dissociation of the appetitive and consummatory phases of feeding behavior in Aplysia: A lesion study. Behav. Biol. 10: 89-97.

Kupfermann, I. (1974b) Feeding behavior in Aplysia: A simple system for the study of motivation. Behav. Biol. 10:1-26.

Kupfermann, I., and T. J. Carew (1974) Behavior patterns of Aplysia californica in its natural environment. Behav. Biol. 12: 317-337.

Kupfermann, I., and J. Cohen (1971) The control of feeding by identified neurons in the buccal ganglion of Aplysia. Am. Zool. 11: 667.

Kupfermann, I., and E. R. Kandel (1969) Neuronal controls of a behavioral response mediated by the abdominal ganglion of Aplysia. Science 164: 847-850.

Kupfermann, I., and H. Pinsker (1968) A behavioral modification of the feeding reflex in Aplysia californica. Commun. Behav. Biol. A 2: 13-17.

Kupfermann, I., and K. R. Weiss (1981) The role of serotonin in arousal of feeding behavior of Aplysia. In Serotonin Neurotransmission and Behavior, A. Gelperin and B. Jacobs, eds., pp. 255-287, M.I.T. Press, Cambridge, MA.

Kupfermann, I., and K. R. Weiss (1982) Activity of an identified serotonergic neuron in free moving Aplysia correlates with behavioral arousal. Brain Res. 241: 334-337.

Laverack, M. S. (1970) Responses of a receptor associated with the buccal mass of Aplysia dactylomela. Comp. Biochem. Physiol. 33: 471-473.

McCaman, R. E., and D. Weinreich (1985) Histaminergic synaptic transmission in the cerebral ganglion of Aplysia. J. Neurophysiol. 53: 1016-1037.

McClellan, A. D. (1982) Movements and motor patterns of the buccal mass of Pleurobranchaea during feeding, regurgitation and rejection. J. Exp. Biol. 98: 195-211.

McCrohan, C. R., and P. R. Benjamin (1980) Synaptic relationships of the cerebral giant cells with motoneurones in the feeding system of Lymnaea stagnalis. J. Exp. Biol. 85: 169-186.

Ono, J. K., and R. E. McCaman (1980) Identification of additional histaminergic neurons in Aplysia: Improvement of single cell isolation techniques for in tandem physiological and chemical studies. Neuroscience $5: 835-840$. 
Pearson, K. G. (1982) Neural circuits for jumping in the locust. Gif lectures in neurobiology: Local neuronal circuits and integration. $J$. Physiol. (Paris) 78: 765-771.

Reingold, S. C., and A. Gelperin (1980) Feeding motor programme in Limax: II. Modulation by sensory inputs in intact animals and isolated central nervous systems. J. Exp. Biol. 85: 1-19.

Roberts, A., N. Dale, and S. R. Soffe (1984) Sustained responses to brief stimuli: Swimming in Xenopus embryos. J. Exp. Biol. 112:321335.

Rose, R. M., and P. R. Benjamin (1979) The relationship of the central motor pattern to the feeding cycle of Lymnaea stagnalis. J. Exp. Biol. 80: 137-163.

Rosen, S. C., K. R. Weiss, J. L. Cohen, and I. Kupfermann (1982) Interganglionic cerebral-buccal mechanoafferents of Aplysia: Receptive fields and synaptic connections to different classes of neurons involved in feeding behavior. J. Neurophysiol. 48: 271-288.

Susswein, A. J., I. Kupfermann, and K. R. Weiss (1976) The stimulus control of biting in Aplysia. J. Comp. Physiol. A 108: 75-96.

Susswein, A. J., K. R. Weiss, and I. Kupfermann (1978) The effects of food arousal on the latency of biting in Aplysia. J. Comp. Physiol. A $123:$ 31-41.

Susswein, A. J., Y. Achituv, M.S. Cappell, D. C. Spray, and M. V. L.
Bennett (1984a) Pharyngeal movements during feeding sequences in Navanax inermis: A cinematographic analysis. J. Comp. Physiol. A 155: 209-218.

Susswein, A. J., S. Gev, Y. Achituv, and S. Markovich (1984b) Behavioral patterns of Aplysia fasciata along the Mediterranean coast of Israel. Behav. Neural Biol. 41: 7-22.

Weiss, K. R., J. L. Cohen, and I. Kupfermann (1978) Modulatory control of buccal musculature by a serotonergic neuron (metacercbral cell) in Aplysia. J. Neurophysiol. 41: 181-203.

Weiss, K. R., U. T. Koch, J. Koester, S. C. Rosen, and I. Kupfermann (1982) The role of arousal in modulating feeding behavior of Aplysia: Neural and behavioral studies. In The Neural Basis of Feeding and Reward, B. G. Hoebel and D. Novin, eds., pp. 25-57, Haer Institute, Brunswick, ME.

Weiss, K. R., E. Shapiro, and I. Kupfermann. (1986a) Modulatory synaptic actions of an identified histaminergic neuron on the serotonergic metacerebral cell of Aplysia. J. Neurosci. 6: 2393-2402.

Weiss, K. R., H. J. Chiel, and I. Kupfermann (1986b). Sensory function and gating of histaminergic neuron C2 in Aplysia. J. Neurosci. 6: 2416-2426.

Wiepkema, P. R. (1971) Positive feedbacks at work during feeding. Behaviour 39: 266-273. 